KYUSHU-HET-94

\title{
New Constraints on SUSY Flavour Mixing in Light of Recent Measurements at the Tevatron
}

\author{
John Foster $^{a 1}$, Ken-ichi Okumura ${ }^{b 2}$ and Leszek Roszkowski ${ }^{c} 3$ \\ a Dipartimento di Fisica, Università di Padova and INFN Sezione di Padova, \\ Via F. Marzolo 8, I-35131, Padua, Italy \\ ${ }^{b}$ Department of Physics, Kyushu University \\ Fukuoka 812-8581, Japan \\ ${ }^{c}$ Department of Physics and Astronomy, University of Sheffield \\ Sheffield S3 7RH, UK
}

\begin{abstract}
We discuss the implications, for general flavour mixing, of the recent results regarding the new allowed range for mixing in the $B_{s}$ meson system, as well as the new improved bounds on $\operatorname{BR}\left(\bar{B}_{s} \rightarrow \mu^{+} \mu^{-}\right)$. Constraints on right handed insertions, in particular, improve considerably in the large $\tan \beta$ regime where the contributions to $B_{s}$ mixing are dominated by double Higgs penguins. Similarly, the allowed regions of parameter space when varying multiple insertions also decrease significantly.
\end{abstract}

\footnotetext{
1 john.foster@pd.infn.it

2 okumura@higgs.phys.kyushu-u.ac.jp

3 L.Roszkowski@sheffield.ac.uk
} 


\section{Introduction}

With the high precision data currently being taken at the B factories and the Tevatron, and the era of the LHC approaching, questions regarding constraining the flavour structure of new physics are now becoming especially pertinent. One model of new physics where the flavour structure can be extremely varied is the Minimal Supersymmetric Standard Model (MSSM). In this model the soft terms that are introduced by supersymmetry (SUSY) breaking can, in principle, be arbitrary matrices in flavour space and lead to potentially catastrophic contributions to well understood processes such as $\bar{B} \rightarrow X_{s} \gamma$. The way this problem is often addressed is to assume that the soft SUSY breaking terms obey the constraints imposed by minimal flavour violation (MFV) [1]. In this framework, the only source of CP and flavour violation in the MSSM is the CKM matrix $K$. While this might initially seem to be rather appealing, it is only an assumption, and it should be confronted with data. Indeed verifying (or falsifying) this assumption would provide a key insight into the possible nature of SUSY breaking.

The means by which one can probe flavour violating effects beyond the MFV scenario is provided by the general flavour mixing (GFM) framework. In GFM the soft terms are treated as being basically arbitrary, with the various flavour violating entries constrained by the currently available experimental data. Variations from MFV are then parameterised by the dimensionless variables $\delta_{X Y}^{d}[2]$

$$
\begin{aligned}
& \left(\delta_{L L}^{d}\right)_{i j}=\frac{\left(m_{d, L L}^{2}\right)_{i j}}{\sqrt{\left(m_{d, L L}^{2}\right)_{i i}\left(m_{d, L L}^{2}\right)_{j j}}}, \\
& \left(\delta_{L R}^{d}\right)_{i j}=\frac{\left(m_{d, L R}^{2}\right)_{i j}}{\sqrt{\left(m_{d, L L}^{2}\right)_{i i}\left(m_{d, R R}^{2}\right)_{j j}}} \\
& \left(\delta_{R L}^{d}\right)_{i j}=\frac{\left(m_{d, R L}^{2}\right)_{i j}}{\sqrt{\left(m_{d, R R}^{2}\right)_{i i}\left(m_{d, L L}^{2}\right)_{j j}}}, \\
& \left(\delta_{R R}^{d}\right)_{i j}=\frac{\left(m_{d, R R}^{2}\right)_{i j}}{\sqrt{\left(m_{d, R R}^{2}\right)_{i i}\left(m_{d, R R}^{2}\right)_{j j}}} .
\end{aligned}
$$

where $i, j=1,2,3$ and $m_{d, X Y}^{2}(X, Y=L, R)$ are related to the conventional soft-terms $m_{Q}^{2}, m_{D}^{2}$, etc., by unitary transformations that rotate the quark fields from the interaction basis to the so-called physical super CKM basis where the quark mass terms are diagonal in flavour space. More details of the notation and conventions we use are presented in [3, 4, 5].

The aim of this Letter is to discuss the new bounds on the flavour violating entries relevant to $b \rightarrow s$ transitions in light of the recent measurements of $B_{s}$ mixing made by the $\mathrm{D} \varnothing$ and CDF Collaborations at the Tevatron [6, 17. Recently the D $\varnothing$ Collaboration has made a preliminary announcement of an upper bound on the parameter $\Delta M_{B_{s}}$ with an allowed range of [6]

$$
17 \mathrm{ps}^{-1}<\left(\Delta M_{B_{s}}\right)_{\mathrm{D} \varnothing}<21 \mathrm{ps}^{-1}
$$

at the $90 \%$ confidence limit. Subsequently the result has been dramatically improved by the CDF Collaboration with the measurement [7]

$$
\left(\Delta M_{B_{s}}\right)_{\mathrm{CDF}}=17.33_{-0.21}^{+0.42} \pm 0.07 \mathrm{ps}^{-1}
$$


where the first error is statistical and the second systematic.

Both results are in reasonable (within $2 \sigma$ ) agreement with the SM predictions resulting either from fits to the unitarity triangle [8]

$$
\left(\Delta M_{B_{s}}\right)_{\text {CKMfitter }}=21.7_{-4.2}^{+5.9} \mathrm{ps}^{-1}, \quad\left(\Delta M_{B_{s}}\right)_{\mathrm{UTfit}}=21.5 \pm 2.6 \mathrm{ps}^{-1},
$$

or from a direct SM calculation [9] (for a discussion of the current status of this prediction and the impact of the various lattice inputs, see [10]). ${ }^{4}$

With the strong evidence for an upper bound on $\Delta M_{B_{s}}$ mixing it is interesting to consider the impact this new constraint will have when constraining general SUSY models.

The supersymmetric corrections to $\Delta M_{B_{s}}$ arise at the one loop level from box diagrams involving the exchange of charginos, gluinos, neutralinos and squarks, as well as contributions arising from charged Higgs exchange [12]. The supersymmetric contributions, which at one loop are the only ones that are sensitive to the flavour structure of SUSY breaking, are typically dominated by the $\alpha_{s}$-enhanced gluino diagrams in the GFM framework. The corrections arising from charginos and neutralinos can therefore be neglected in regions of parameter space where $\tan \beta$ is small. However, as $\tan \beta$ increases, the two contributions can become increasingly important and must be taken into account in a consistent analysis of the bounds on the insertions. In addition to these diagrams, at large $\tan \beta$ a new two loop contribution arises due to double Higgs penguins mediated by neutral Higgs [13, 14, 15, 16, 4]. These contributions benefit from an enhancement by $\tan ^{4} \beta$ and vary only as $1 / m_{A}^{2}$ (the mass of the pseudoscalar Higgs $A^{0}$ ). When compared to the $1 / m_{\widetilde{q}}^{2}$ dependence (where $m_{\widetilde{q}}$ is the squark mass scale) of the SUSY mediated box diagrams it is apparent that, when $\tan \beta$ is large, the double Higgs penguin contributions can play a significant rôle and even dominate the dynamics associated with the new physics contributions to $B_{s}$ mixing.

Of course, $B_{s}$ mixing is not the only possible constraint on the supersymmetric contributions to $b \rightarrow s$ transitions. As discussed in [4, 5] both $\bar{B} \rightarrow X_{s} \gamma$ and $\bar{B}_{s} \rightarrow \mu^{+} \mu^{-}$can also play a rôle in this endeavour. The decay $\bar{B} \rightarrow X_{s} \gamma$, for instance, has remained a mainstay in analyses of $b \rightarrow s$ transitions for some time now and provides quite stringent bounds on certain sources of flavour violation in the MSSM [17, 3, 4]. The decay $\bar{B}_{s} \rightarrow \mu^{+} \mu^{-}$, on the other hand, currently remains unobserved, however it is intimately linked to $B_{s}$ mixing as both processes can benefit from large enhancement through neutral Higgs penguin dynamics in the large $\tan \beta$ regime. The neutral Higgs penguin contribution to $\bar{B}_{s} \rightarrow \mu^{+} \mu^{-}$benefits from $\tan ^{6} \beta$ enhancement [13, 14, 15, 16, 4] and, due to the helicity suppression of the SM contribution it is possible to induce exceptionally large contributions to the decay that can quite easily approach the preliminary limit from the CDF Collaboration [18, of

$$
\operatorname{BR}\left(\bar{B}_{s} \rightarrow \mu^{+} \mu^{-}\right)_{\mathrm{CDF}}<8.0 \times 10^{-8}
$$

at the $90 \%$ confidence level. The value of the bound increases to $1.0 \times 10^{-7}$ at the $95 \%$ confidence level. For reference the SM prediction for the branching ratio is [19]

$$
\mathrm{BR}\left(\bar{B}_{s} \rightarrow \mu^{+} \mu^{-}\right)_{\mathrm{SM}}=(3.46 \pm 1.5) \times 10^{-9} .
$$

\footnotetext{
${ }^{4}$ Using the lattice inputs described in [11] we obtain a central value of $18.9 \mathrm{ps}^{-1}$ for the SM contribution that is used in the forthcoming plots.
} 
As both $B_{s}$ mixing and $\bar{B}_{s} \rightarrow \mu^{+} \mu^{-}$share a similar dependence on the same underlying vertex, it is natural to ask how the new bound on $B_{s}$ mixing might effect the current prospects for the detection of $\bar{B}_{s} \rightarrow \mu^{+} \mu^{-}$.

In this Letter we shall perform a complete analysis of the constraints that emerge from the new CDF bound on $\Delta M_{B_{s}}$ (44). To ensure the validity of our analysis in as wide a range of parameter space as possible we shall compute the contributions arising from all the possible SUSY contributions to $B_{s}$ mixing that arise at the one loop level as well as the double Higgs penguin diagram that becomes significant at large $\tan \beta$.

In addition, we apply the procedure discussed in detail in [4] to include the large effects that appear beyond the leading order (BLO). These effects include terms that are enhanced by either large logs or $\tan \beta$ [20, 15, 16, 3, 4. Large logs are induced by running from the SUSY scale $\mu_{S U S Y}$ to the electroweak scale $\mu_{W}$. The terms enhanced by $\tan \beta$, on the other hand, arise from threshold corrections to the down quark mass matrix [21, 4] and Higgs vertices [22, 20, 14, 13, 15, 16, 4]. The $\tan \beta$ enhanced terms can be especially important, and their inclusion can lead to large differences between a purely LO calculation and one that properly takes into account BLO effects [3, 4]. In particular, the inclusion of such BLO corrections leads to a focusing effect that, at large $\tan \beta$ and $\mu>0$, can significantly loosen the bounds on SUSY sources of flavour violation [3, 4.

Other recent analyses have also discussed the impact of the recent measurement (44) both in the context of MFV 23] and GFM [24, 10]. We should point out, however, that, in contrast to the analyses presented in 24, 10, we take into account all the relevant SUSY one-loop diagrams as well as the double Higgs penguin diagram that appears at the two-loop level. Possible enhancements to the phase associated with $B_{s}$ mixing have also been discussed within the context of this new constraint [25]. In this analysis, however, we shall treat the various sources of SUSY flavour violation as real and any contributions to the phase of $B_{s}$ mixing would therefore be expected to be small in this limit.

The constraints we impose take the following form. For $\bar{B} \rightarrow X_{s} \gamma$ we require that the prediction for $\operatorname{BR}\left(\bar{B} \rightarrow X_{s} \gamma\right)$ lies between the bounds

$$
2.65 \times 10^{-4}<\operatorname{BR}\left(\bar{B} \rightarrow X_{s} \gamma\right)<4.35 \times 10^{-4},
$$

a range that can be obtained by adding the SM and experimental errors in quadrature and taking the $2 \sigma$ interval around the most recent experimental world average of $\left(3.55 \pm 0.24_{-0.10}^{+0.09} \pm 0.03\right) \times 10^{-4}[26$ ] (compared to $(3.57 \pm 0.30) \times 10^{-4}$ in the $\left.\mathrm{SM}[27]\right)$. For $\bar{B}_{s} \rightarrow \mu^{+} \mu^{-}$we require that $\operatorname{BR}\left(\bar{B}_{s} \rightarrow \mu^{+} \mu^{-}\right)$ satisfies the $90 \%$ confidence interval ([6) and, finally, for $\Delta M_{B_{s}}$ we impose the constraint

$$
12.53 \mathrm{ps}^{-1}<\Delta M_{B_{s}}<22.13 \mathrm{ps}^{-1}
$$

with the allowed range obtained by taking the $1 \sigma$ interval after combining the experimental and $\mathrm{SM}$ errors in quadrature. This requirement, in particular is especially strict, however, it provides an appreciation of the possible implications of the new results concerning $B_{s}$ mixing.

Finally, while we parameterise the amount of flavour violation using $\delta_{X Y}^{d}$ we should emphasise that all of the forthcoming calculations are performed in the mass eigenstate basis, rather than the mass insertion approximation that can become inaccurate for large values of $\delta_{X Y}^{d}$. 


\section{Constraints on Single Insertions}

Let us first consider the constraints that can be placed on single insertions. As discussed in the previous section, the contributions to $\Delta M_{B_{s}}$ in the large $\tan \beta$ regime are dominated by double Higgs penguins that benefit from an enhancement by $\tan ^{4} \beta$, as well as a reduced mass suppression, compared to the gluino mediated box diagrams that are the dominant contribution at low $\tan \beta$. Using the results concerning the neutral Higgs penguin gathered in [13, 15, 16, 4] it is relatively easy to evaluate the contributions to $\Delta M_{B_{s}}$ arising from these diagrams

$$
\begin{aligned}
\Delta M_{B_{s}}^{D P}= & -\frac{8 G_{F}}{3 \sqrt{2}} m_{B_{s}} f_{B_{s}}^{2} P_{2}^{L R} \frac{m_{b}^{2} \tan ^{4} \beta}{m_{A}^{2}} \\
& \times\left\{\frac{\left(\epsilon_{Y} Y_{t}^{2} K_{t s} K_{t b}^{*}+\epsilon_{L L} x_{\widetilde{d}_{L}} \delta_{L L}^{d}\right)}{\left(1+\epsilon_{3} \tan \beta\right)\left(1+\epsilon_{s} \tan \beta\right)}+\frac{m_{\widetilde{g}}}{m_{b}} \frac{\epsilon_{R L} \epsilon_{s} x_{\widetilde{d}_{R L}} \delta_{L R}^{d}}{\left(1+\epsilon_{s} \tan \beta\right)}+\frac{m_{s}}{m_{b}} \frac{\epsilon_{R R} x_{\widetilde{d}_{R}} \delta_{R R}^{d}}{\left(1+\epsilon_{s} \tan \beta\right)^{2}}\right\} \\
& \times\left\{\frac{m_{s}}{m_{b}} \frac{\left(\epsilon_{Y} Y_{t}^{2} K_{t s} K_{t b}^{*}+\epsilon_{L L} x_{\widetilde{d}_{L}} \delta_{L L}^{d}\right)}{\left(1+\epsilon_{3} \tan \beta\right)\left(1+\epsilon_{s} \tan \beta\right)}+\frac{m_{\widetilde{g}}}{m_{b}} \frac{\epsilon_{R L} \epsilon_{3} x_{\widetilde{d}_{R L}} \delta_{R L}^{d}}{\left(1+\epsilon_{3} \tan \beta\right)}+\frac{\epsilon_{R R} x_{\widetilde{d}_{R}} \delta_{R R}^{d}}{\left(1+\epsilon_{3} \tan \beta\right)^{2}}\right\},
\end{aligned}
$$

where $f_{B_{s}}$ and $P_{2}^{L R}$ (see 28] for a definition) are determined from the lattice and are the principle source of error associated with the calculation for $\Delta M_{B_{s}}$. Aside from $\tan \beta$ and $m_{A}$ the various SUSY parameters only enter as ratios encoded in the factors of $\epsilon$ that are gathered in the appendix. This fact gives rise to a non-decoupling effect where the double penguin contribution to $\Delta M_{B_{s}}$ can be sizable, even for multi- $\mathrm{TeV}$ scale sparticle masses, provided that the Higgs sector remains relatively light. The BLO corrections to the above expression take the form of the factors of $1+\epsilon_{3} \tan \beta$ and $1+\epsilon_{s} \tan \beta$ that appear in the denominators of each term. For $\mu>0(\mu<0)$ these factors typically act to suppress (enhance) the double Higgs penguin contribution. In addition to these corrections the insertions $\delta_{L R}^{d}$ and $\delta_{R L}^{d}$ also appear in the above expression once BLO effects are taken into account 4 .

Numerically, for degenerate sparticle parameters of $1 \mathrm{TeV}$ the contribution to $\Delta M_{B_{s}}$ becomes

$$
\begin{aligned}
\Delta M_{B_{s}}^{D P}= & -18 \mathrm{ps}^{-1}\left(\frac{f_{B_{s}}}{230 \mathrm{MeV}}\right)^{2}\left(\frac{P_{2}^{L R}}{2.56}\right)\left(\frac{\tan \beta}{40}\right)^{4}\left(\frac{500 \mathrm{GeV}}{m_{A}}\right)^{2} \\
& \times\left\{\frac{\left(1-33.6 \delta_{L L}^{d}\right)}{\left(1 \pm 0.38 \frac{\tan \beta}{40}\right)\left(1 \pm 0.29 \frac{\tan \beta}{40}\right)}+\frac{385.2 \delta_{L R}^{d}}{\left(1 \pm 0.38 \frac{\tan \beta}{40}\right)}-\frac{0.75 \delta_{R R}^{d}}{\left(1 \pm 0.29 \frac{\tan \beta}{40}\right)^{2}}\right\} \\
& \times\left\{\frac{0.022\left(1-33.6 \delta_{L L}^{d}\right)}{\left(1 \pm 0.38 \frac{\tan \beta}{40}\right)\left(1 \pm 0.29 \frac{\tan \beta}{40}\right)}+\frac{290.6 \delta_{R L}^{d}}{\left(1 \pm 0.29 \frac{\tan \beta}{40}\right)}-\frac{33.6 \delta_{R R}^{d}}{\left(1 \pm 0.29 \frac{\tan \beta}{40}\right)^{2}}\right\},
\end{aligned}
$$

where we have assumed that the trilinear soft mass term $A_{u}$ has the opposite sign compared to $\mu$ to ensure compatibility with the constraint supplied by $\bar{B} \rightarrow X_{s} \gamma$. The sign of the contributions that appear in the denominator of each term is determined by $\operatorname{sgn}(\mu)$. It should be noted that 
the seemingly large contributions arising from LR and RL insertions to each Higgs penguin are tempered by the fact that these insertions scale as $1 / M_{\text {SUSY }}$ and are, therefore, at least one or two orders of magnitude smaller than LL and RR insertions.

Inspection of Eqs. (10)-(11) reveals that in the limit of MFV, or when only an LL or LR insertion is non-zero, the double Higgs penguin contribution to $\Delta M_{B_{s}}$ is suppressed by $m_{s} / m_{b}$. In addition, the contributions to $\Delta M_{B_{s}}$ arising from MFV effects and LL insertions destructively interfere with the SM value driving it towards the current lower bound on the parameter [13, 15, 16. As such, the impact of the new upper bound in constraining both MFV and LL or LR insertions tends to be negligible once one takes into account the constraints arising from $\bar{B} \rightarrow X_{s} \gamma$ and $\bar{B}_{s} \rightarrow \mu^{+} \mu^{-}$.

It is evident from the above formulae, however, that GFM effects allow one to avoid the suppression by $m_{s} / m_{b}$ in the presence of either RL or RR insertions. If only these insertions are non-zero (see the next section for a discussion regarding the more general case) the suppression by the strange quark mass is overcome via the interplay between the MFV contribution to one of the neutral Higgs penguins and the contribution arising from either one of these insertions. This effect, which was first pointed out in [4], can give to rise to extremely large contributions to $\Delta M_{B_{s}}$ while still being in agreement with the current bounds on $\bar{B}_{s} \rightarrow \mu^{+} \mu^{-}$and $\bar{B} \rightarrow X_{s} \gamma$. In addition, the sign of the correction to $\Delta M_{B_{s}}$ depends on the sign of the RL or RR insertion. Consequently, it is possible to generate contributions to $B_{s}$ mixing that interfere constructively with the SM result and any upper bound on $B_{s}$ mixing will, therefore, provide a useful constraint on these two insertions in the large $\tan \beta$ regime. One should note that such a contribution would be absent in a simplistic analysis in which only GFM effects arising from gluinos were taken into account. It is therefore vital to properly address the contributions arising from electroweak (EW), and therefore MFV, effects arising from higgsino exchange in any analysis placing bounds on these two insertions in the large $\tan \beta$ regime.

Finally, let us point out that the formulae in Eqs. (10)-(11) are only approximate and do not include, for instance, the additional EW corrections arising from gaugino exchange, which can, in some instances, have up to a $20 \%$ effect 15, 4. All such corrections are taken into account in our numerical analysis.

Before discussing the parameter dependence of the new bounds on $B_{s}$ mixing and $\bar{B}_{s} \rightarrow \mu^{+} \mu^{-}$ let us briefly comment on the correlation between the two observables. Such a situation is illustrated in Fig. 1 where, in the panel on the left, we show the correlation between the $\operatorname{BR}\left(\bar{B}_{s} \rightarrow \mu^{+} \mu^{-}\right)$ and $\Delta M_{B_{s}}$ for varying $\delta_{R R}^{d}$ (one can find a similar plot for $\delta_{L L}^{d}$ insertions in Fig. 26 of [4]). As is evident from the plot, contributions to $\bar{B}_{s} \rightarrow \mu^{+} \mu^{-}$arising from these insertions are now severely constrained by the new bounds on $B_{s}$ mixing. It is well known that varying all four insertions at the same time essentially destroys a large amount of the correlation between the two variables and this is illustrated in the figure to the right. However, it is also apparent that both bounds on $B_{s}$ mixing and $\operatorname{BR}\left(\bar{B}_{s} \rightarrow \mu^{+} \mu^{-}\right)$rule out a large proportion of the available parameter space in this scenario. We shall discuss the limits on multiple insertions in the next section.

Fig. 2 shows the $\tan \beta$ dependence of the new constraints arising from the new upper bound on $B_{s}$ mixing as well as the improved bound on $\bar{B}_{s} \rightarrow \mu^{+} \mu^{-}$(6). The top two panels of the figure illustrate the bounds imposed on $\delta_{L L}^{d}$ and $\delta_{L R}^{d}$ insertions. In these two cases the effect of the new bound on $B_{s}$ mixing is relatively minor. The constraint on $\delta_{L L}^{d}$ insertions, for instance, only eliminates an extreme region of parameter space at low $\tan \beta$ where the amplitude for $\bar{B} \rightarrow X_{s} \gamma$ 

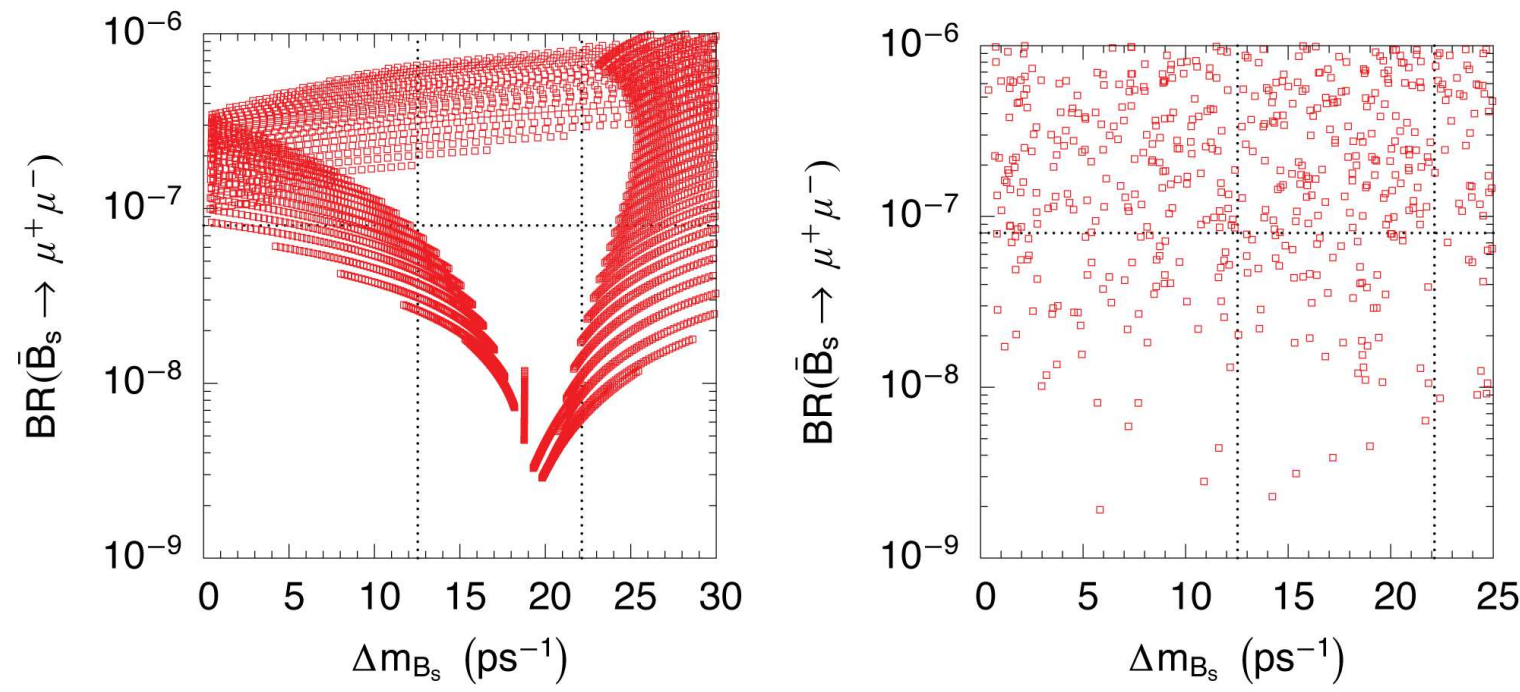

Figure 1: Scatter plots illustrating the correlation between $\operatorname{BR}\left(\bar{B}_{s} \rightarrow \mu^{+} \mu^{-}\right)$and $\Delta M_{B_{s}}$. In the panel to the left the insertion $\delta_{R R}^{d}$ is scanned over the interval $[-0.8,0.8]$ and $m_{\widetilde{q}}$ is varied over the range $[500,1500] \mathrm{GeV}$ the remaining parameters are given by $\mu=m_{A}=-A_{u}=500 \mathrm{GeV}$, $m_{\widetilde{g}}=1 \mathrm{TeV}$. In the panel on the right all four insertions are varied, with $\delta_{L L}^{d}$ and $\delta_{R R}^{d}$ over the range $[-0.8,0.8]$, and $\delta_{L R}^{d}$ and $\delta_{R L}^{d}$ over the range $[-0.08,0.08]$. The soft sector is described by $m_{\widetilde{q}}=-A_{u}=m_{\widetilde{g}} / \sqrt{2}=\sqrt{2} \mu=2 m_{A}=1 \mathrm{TeV}$. In both panels $\tan \beta=40$. Points inconsistent with the constraint supplied by $\bar{B} \rightarrow X_{s} \gamma$ are not shown.

has effectively flipped sign, while for LR insertions that constraint has no effect on the bounds whatsoever. From these two panels it is also possible to appreciate the effect that improving the bound on $\bar{B}_{s} \rightarrow \mu^{+} \mu^{-}$might have when constraining the insertions. For instance in both panels it is possible to see that improving the bound to $2 \times 10^{-8}$ (a conservative value that is achievable at the Tevatron) would start playing an important role in constraining the insertions at large $\tan \beta$. A bound of $5 \times 10^{-9}$ (thereby ruling out any large new physics contributions to $\bar{B}_{s} \rightarrow \mu^{+} \mu^{-}$) would provide the bounds on the insertions for values of $\tan \beta$ as low as 10 .

As discussed in [4, 5], the bounds on the insertions $\delta_{R L}^{d}$ and $\delta_{R R}^{d}$ are far more dependent on the $\Delta M_{B_{s}}$ constraint, and this is evident in the two lower panels. For both insertions the bounds at large $\tan \beta$ are now imposed entirely by the $B_{s}$ mixing constraint. In fact, for RR insertions, the bounds attributable to the $\Delta M_{B_{s}}$ constraint are present for values of $\tan \beta$ as low as 10 (for $\left.\delta_{R R}^{d}>0\right)$ while values of $\mathcal{O}(1)$ for the insertion are now heavily disfavoured in the large $\tan \beta$ regime. It is also evident from the plots that improving the limit on $\bar{B}_{s} \rightarrow \mu^{+} \mu^{-}$will have relatively little effect on the possible bounds that can be placed on the two insertions until values approaching the SM prediction are reached. The flip side of this argument is, of course, that if the Tevatron were to measure $\bar{B}_{s} \rightarrow \mu^{+} \mu^{-}$in the region of $3-10 \times 10^{-8}$ one could almost automatically rule out large contributions from RR or RL insertions being responsible for such an enhancement (ignoring possible fine tuned scenarios). 

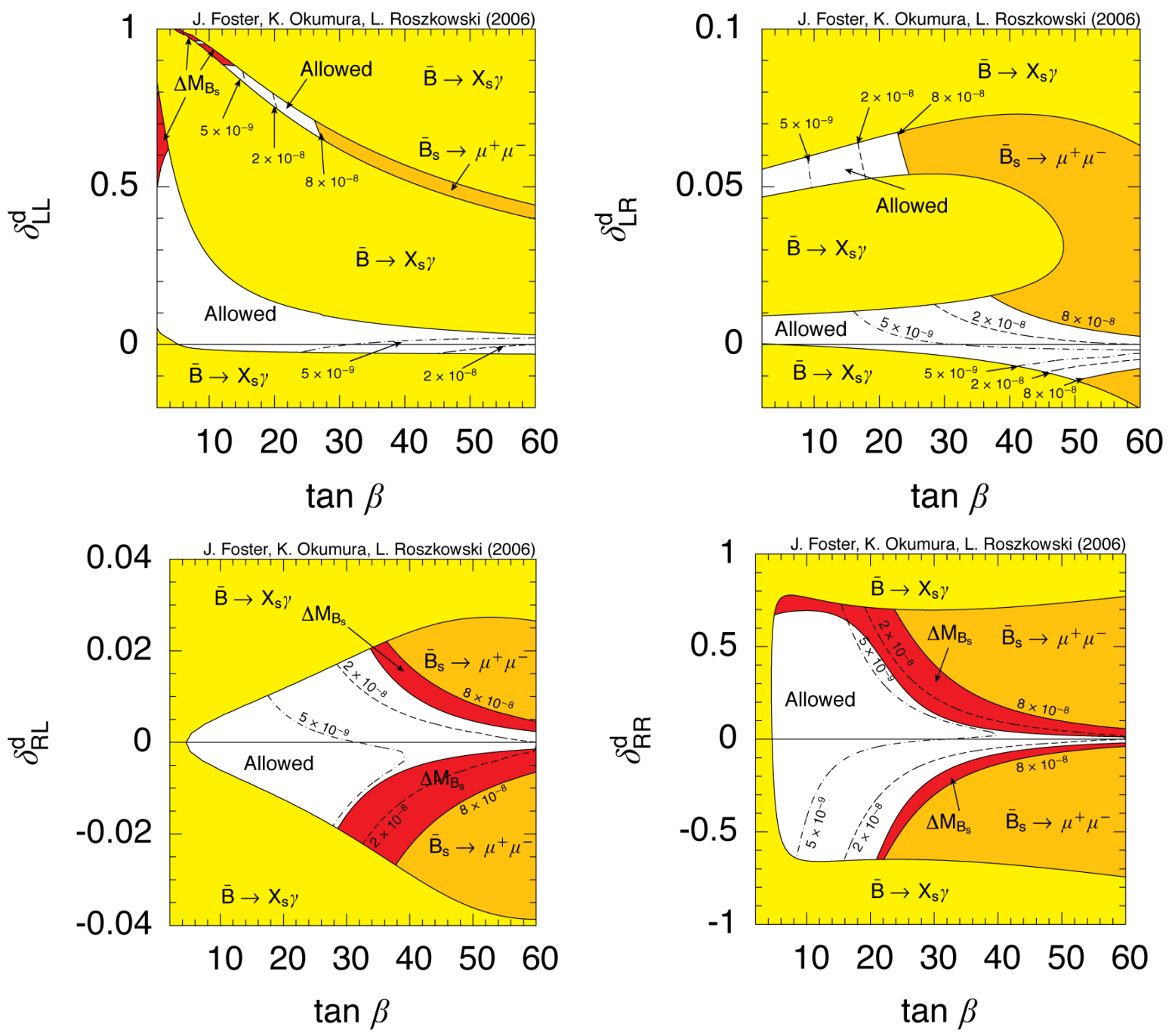

Figure 2: Contour plots showing the limits on the GFM parameters $\delta_{X Y}^{d}$ for varying $\tan \beta$. In each panel only one $\delta_{X Y}^{d}$ is varied and the rest are put to zero. The soft sector is parameterised as follows $m_{\widetilde{q}}=m_{\widetilde{g}}=1 \mathrm{TeV}$ and $\mu=-A_{u}=500 \mathrm{GeV}$ and $m_{A}=500 \mathrm{GeV}$. Regions excluded by $\bar{B} \rightarrow X_{s} \gamma$ (i.e. outside of the region $\left.2.65 \times 10^{-4}<\mathrm{BR}\left(\bar{B} \rightarrow X_{s} \gamma\right)<4.35 \times 10^{-4}\right)$ are shaded in yellow (light grey). The subsequent regions that are excluded by the CDF limit of $8 \times 10^{-8}$ on $\bar{B}_{s} \rightarrow \mu^{+} \mu^{-}$are shaded in orange (medium grey). The remaining regions that are excluded by the $\mathrm{D} \varnothing$ and CDF results on $\Delta M_{B_{s}}$ are shaded in red (dark grey). It should be noted that we relax the allowed region to $12.53 \mathrm{ps}^{-1}<\Delta M_{B_{s}}<22.13 \mathrm{ps}^{-1}$ to take into account the errors associated with the lattice inputs required to evaluate $\Delta M_{B_{s}}$. Finally, contours depicting values of $2 \times 10^{-8}$ and $5 \times 10^{-9}$ for $\operatorname{BR}\left(\bar{B}_{s} \rightarrow \mu^{+} \mu^{-}\right)$are shown and are delineated by dashed and dot-dashed lines respectively. 

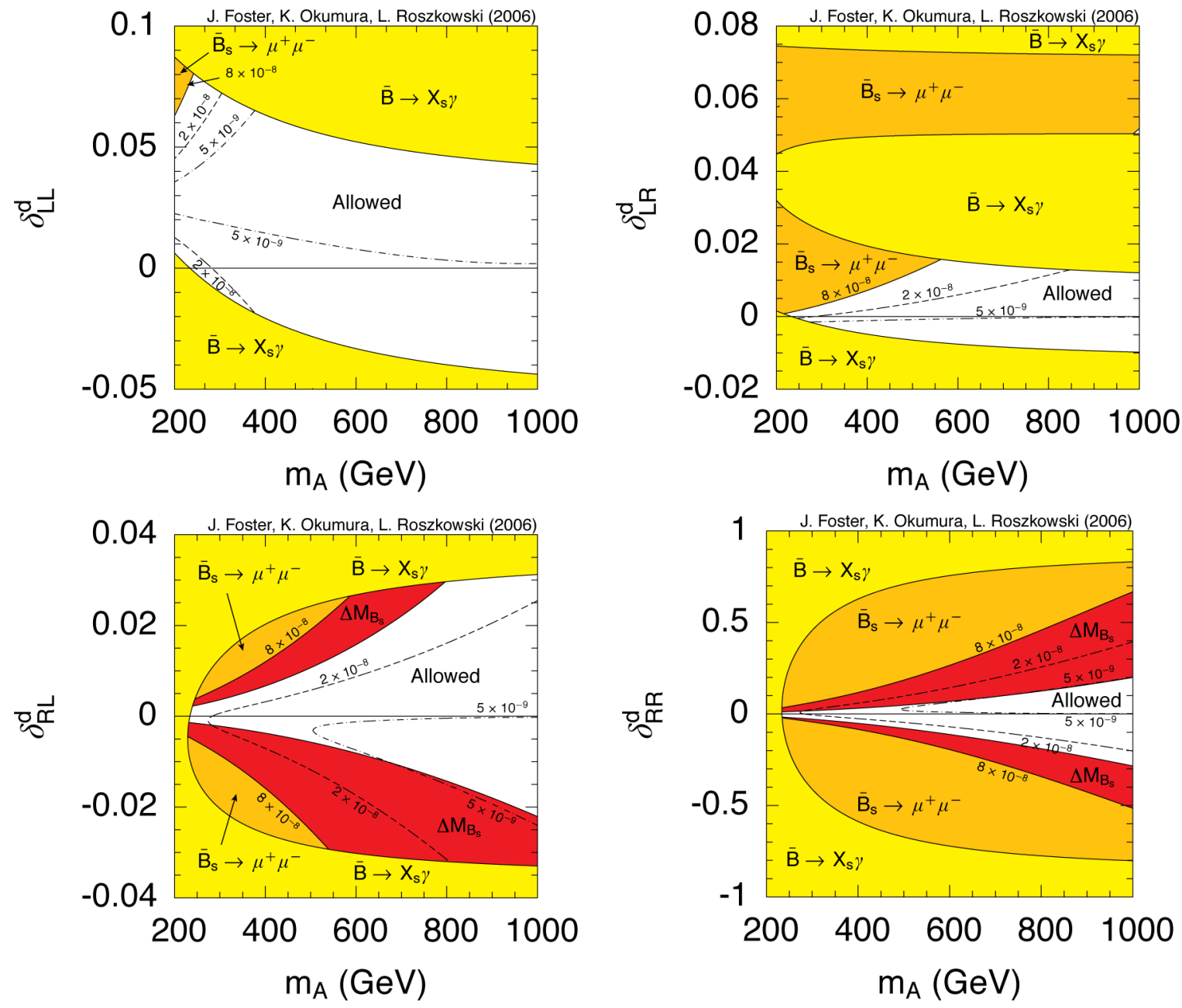

Figure 3: Contour plots showing the limits on the GFM parameters $\delta_{X Y}^{d}$ for varying $m_{A}$. The soft sector is parameterised as follows $m_{\widetilde{q}}=m_{\widetilde{g}}=1 \mathrm{TeV}$ and $\mu=-A_{u}=500 \mathrm{GeV}$ and $\tan \beta=40 \mathrm{GeV}$. The excluded and allowed regions are shaded in a similar manner to Fig. 2

As the constraint supplied by $B_{s}$ mixing also depends on $m_{A}$, it is natural to ask how the bounds on the insertions change when this parameter is varied. Such a scenario is illustrated in Fig. 3. In the top two panels illustrating the bounds on LL and LR insertions we see, once again, that the new bound on $B_{s}$ mixing has no effect on the bounds that can be placed on the two insertions when compared to the existing constraints supplied by the $\Delta F=1$ processes $\bar{B} \rightarrow X_{s} \gamma$ and $\bar{B}_{s} \rightarrow \mu^{+} \mu^{-}$. On the other hand, the impact of improving the bound on $\bar{B}_{s} \rightarrow \mu^{+} \mu^{-}$is more substantial. It is clear that any prospective improvements will affect the low $m_{A}$ region of parameter space and improve the possible bounds on the insertions substantially.

The lower two panels illustrate the bounds on the insertions $\delta_{R L}^{d}$ and $\delta_{R R}^{d}$. Once again, we can 

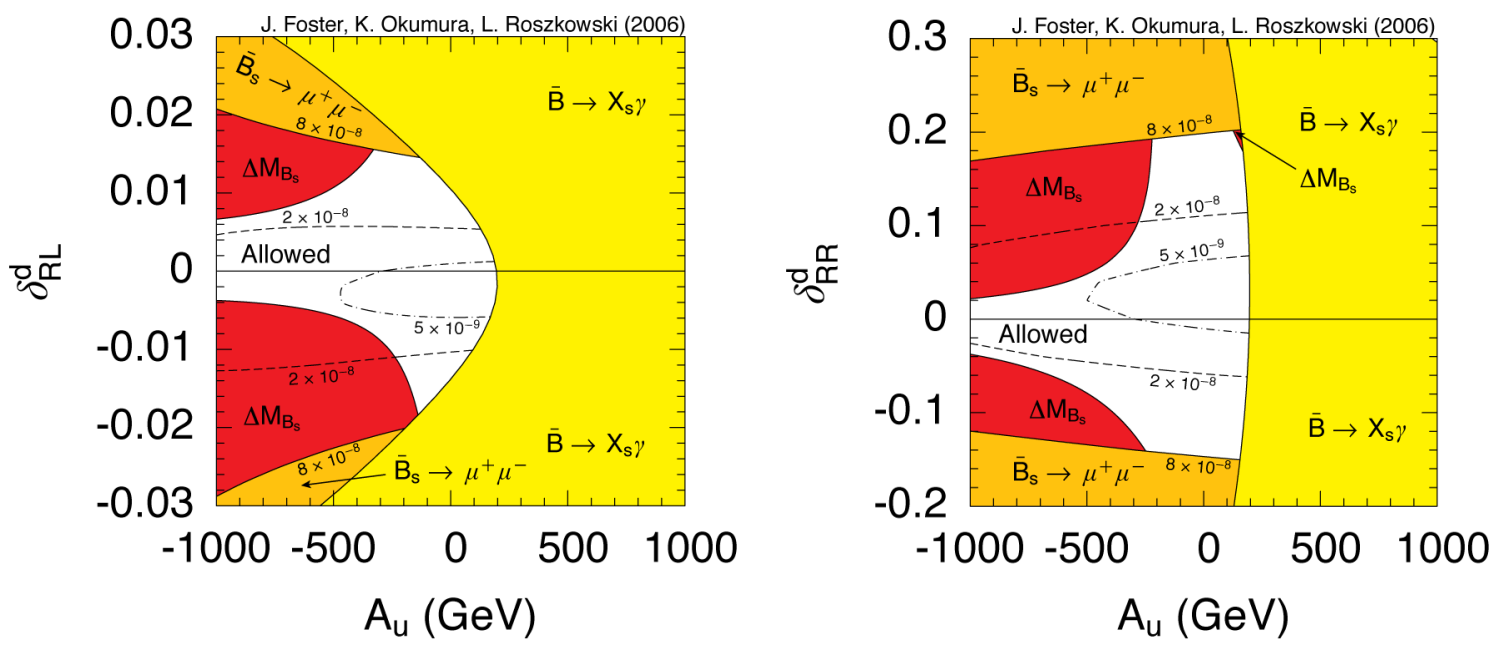

Figure 4: Contour plots showing the allowed ranges for $\delta_{R L}^{d}$ and $\delta_{R R}^{d}$ for varying $A_{u}$. The soft sector is parameterised as follows $m_{\widetilde{q}}=m_{\widetilde{g}}=1 \mathrm{TeV}$ and $\mu=m_{A}=500 \mathrm{GeV}$ and $\tan \beta=40 \mathrm{GeV}$. The excluded and allowed regions are shaded in a similar manner to Fig. 2

see the dramatic effect the constraint supplied by $B_{s}$ mixing has. Even when $m_{A}$ is of a similar order as the squark masses (i.e. $1 \mathrm{TeV}$ ) it is clear that useful bounds can be placed on both insertions, that surpass those derived from either $\bar{B}_{s} \rightarrow \mu^{+} \mu^{-}$or $\bar{B} \rightarrow X_{s} \gamma$. In a similar manner to the plots that appeared in Fig. 2 the effect of improving the bound on $\bar{B}_{s} \rightarrow \mu^{+} \mu^{-}$to $2-3 \times 10^{-8}$ on the constraints on the two insertions will be relatively minor, however improving the limit to $5 \times 10^{-9}$ would prove to be most useful in further constraining the allowed flavour violation in the RL and RR sectors.

It is natural to consider how varying other parameters might affect the bounds one can place on these insertions. As is evident from (10) the remaining SUSY parameters enter as ratios encoded in the factors of $\epsilon_{i}$. Therefore universally scaling the SUSY spectrum will have relatively little effect on the bounds, provided that $m_{A}$ and $\tan \beta$ remain constant. The dependence on single parameters such as $\mu$ and $A_{u}$ can, however, be more complex.

Varying the $\mu$ parameter, for instance, generally has the effect of increasing or decreasing the magnitude of the factors of $\epsilon$ that appear in (10). Increasing $\mu$ initially strengthens the bounds one can place on the insertions using $\bar{B}_{s} \rightarrow \mu^{+} \mu^{-}$or $B_{s}$ mixing, however, once it reaches a similar order of magnitude as $m_{\tilde{g}}$ or $m_{\widetilde{q}}$ the bounds tend to remain relatively independent of further variations in the parameter.

The effect of varying the parameter $A_{u}$ is illustrated in Fig. 4. $A_{u}$ enters into the higgsino contribution to the neutral Higgs penguin and is encoded in the factor of $\epsilon_{Y}$ that appears in (10). As such its effect on the bounds imposed on LL or LR insertions tends to be rather small. For $\mathrm{RL}$ and RR insertions, on the other hand, the effect of varying the parameter can be appreciably larger, as $\epsilon_{Y}$ (and therefore $A_{u}$ ) enters into the double Higgs penguin contribution arising from these insertions to $\Delta M_{B_{s}}$. Both panels in Fig. [4 illustrate that increasing the magnitude of $A_{u}$ tends to 
increase the effectiveness of the $B_{s}$ mixing bound when constraining $\delta_{R L}^{d}$ and $\delta_{R R}^{d}$ insertions. Indeed it can be seen from both plots that, when $A_{u}$ is small, the constraints on the insertions are typically replaced by those arising from the decay $\bar{B}_{s} \rightarrow \mu^{+} \mu^{-}$. It should also be noted that the bounds in each figure are relatively independent of the sign of $A_{u}$, except those arising from $\bar{B} \rightarrow X_{s} \gamma$ that tend to favour $A_{u}<0$ if $\mu>0$ and therefore rule out large, positive values of $A_{u}$ in both plots.

\section{Constraints on Multiple Insertions}

As $B_{s}$ mixing is a $\Delta F=2$ process it is naturally more sensitive to scenarios where more than one insertion is present at a given time. It is therefore useful to consider how the new results regarding $B_{s}$ mixing will affect the bounds on multiple sources of flavour violation. If one refers to the formula given in (10) in the previous section, it can be seen that exceptionally large contributions to $B_{s}$ mixing are possible when an LL or an LR insertion is varied at the same time as a RL or RR insertion. These large contributions to $B_{s}$ mixing can easily be in conflict with current limits provided by DØ (3) and CDF (4) and they will, therefore, provide an excellent constraint in such scenarios. Such a situation is illustrated in Fig. 5 where we show the six possible combinations that can be formed when varying two insertions at a time.

From the figure it can be seen immediately that the new bound imposed by $B_{s}$ mixing has a substantial effect on the available parameter space in all but one of the six panels. (The $\delta_{L L}^{d}-\delta_{L R}^{d}$ parameter space is left relatively unaffected by the $B_{s}$ mixing constraint as the corresponding contribution to $\Delta M_{B_{s}}$ is suppressed by $m_{s} / m_{b}$.) In fact, in the four panels constraining the combination of an LL or LR insertion together with an RL or RR insertion it can be seen that the constraints on the available parameter space are almost completely dominated by $B_{s}$ mixing with the additional constraints supplied by either $\bar{B} \rightarrow X_{s} \gamma$ or $\bar{B}_{s} \rightarrow \mu^{+} \mu^{-}$only ruling out regions where either RL or RR insertions are small, or fine tuned regions where an LL or LR insertion accidentally cancels with the MFV contribution to the neutral Higgs penguin.

Also illustrated in all six panels is the effect that an improvement in the determination of the upper bound on the branching ratio for $\bar{B}_{s} \rightarrow \mu^{+} \mu^{-}$might have. From all of the panels it is evident that improving the limit to $2 \times 10^{-8}$ will further reduce the available parameter space. However, in all but the top left panel it is also apparent that the lower and upper bounds on $B_{s}$ mixing play a far more important role in constraining certain combinations of the insertions. Indeed, in much of the parameter space, only after the limit on $\bar{B}_{s} \rightarrow \mu^{+} \mu^{-}$has reached a level approaching $5 \times 10^{-9}$ would it provide a bound exceeding that imposed by $B_{s}$ mixing when constraining multiple pairs of insertions.

\section{Conclusions}

In this Letter we have summarised the new constraints that can be placed on SUSY flavour violation in light of the recent limits placed on $\Delta M_{B_{s}}$ and the improved bound on $\bar{B}_{s} \rightarrow \mu^{+} \mu^{-}$at the Tevatron. While unfortunately this observation has not signalled the presence of physics beyond the Standard Model, it now places strict new constraints on SUSY flavour violation. In particular, RR and RL insertions are now constrained to a similar degree as LL and LR insertions in the large $\tan \beta$ 

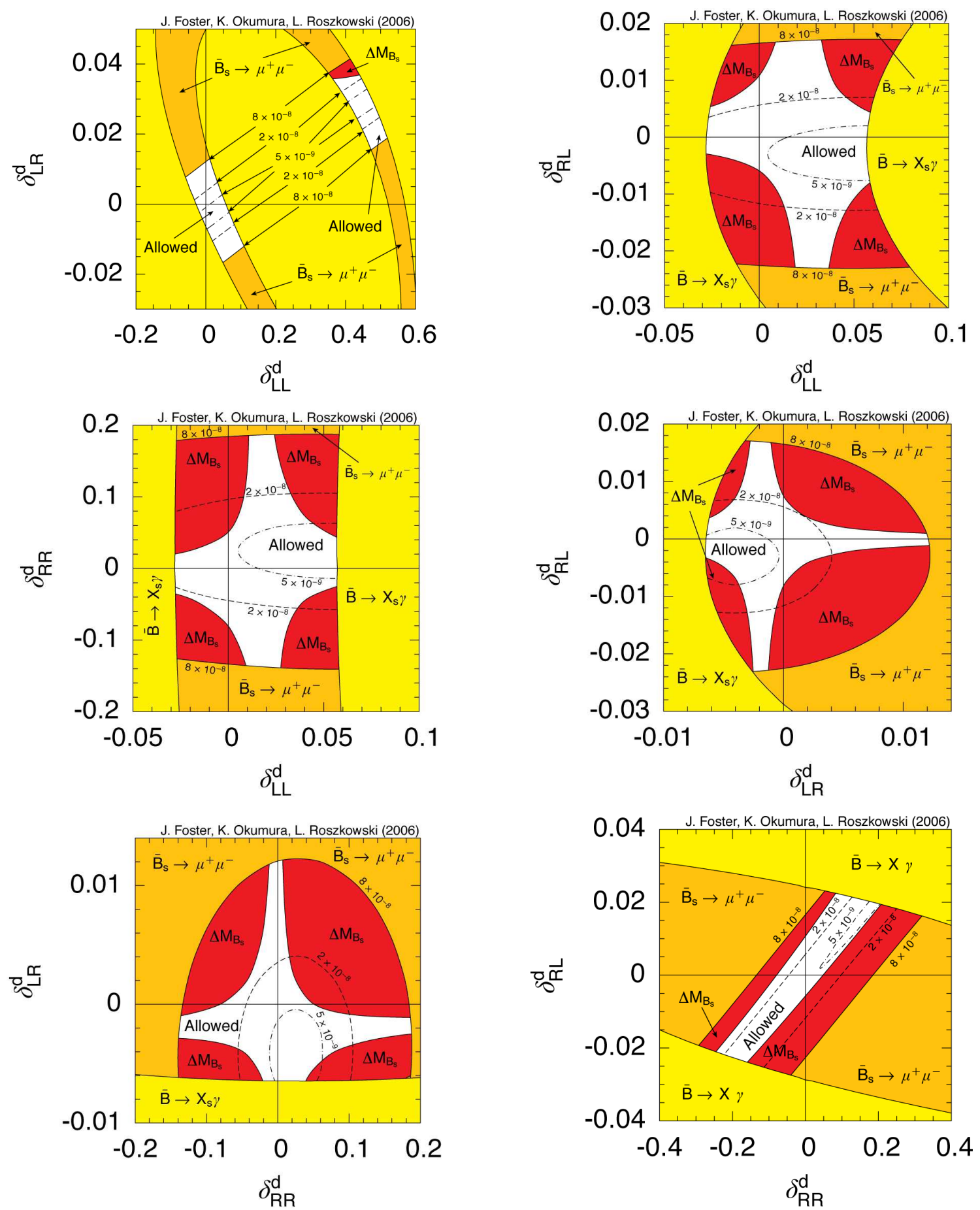

Figure 5: The soft sector is parameterised as follows $m_{\widetilde{q}}=m_{\tilde{g}}=1 \mathrm{TeV}$ and $m_{A}=\mu=-A_{u}=$ $500 \mathrm{GeV}$ and $\tan \beta=40 \mathrm{GeV}$. The excluded and allowed regions are shaded in a similar manner to Fig. 2 . 
regime. Consequently models that predict large values for these insertions (such as grand unified theories that incorporate a SUSY seesaw model, for instance [29]) might now encounter strict constraints in the large $\tan \beta$ regime unless the Higgs sector is naturally very heavy (i.e. $m_{A} \gg 1 \mathrm{TeV}$ ). In addition, this observation seems to rule out large contributions to $\bar{B}_{s} \rightarrow \mu^{+} \mu^{-}$arising from RR insertions and any forthcoming measurement of $\bar{B}_{s} \rightarrow \mu^{+} \mu^{-}$at the Tevatron would be hard to reconcile with the effect of $\mathrm{RR}$ insertions unless $\left|A_{u}\right|$ is small.

\section{Acknowledgements}

We would like to thank A. Masiero for helpful comments. This work was partially supported by the EC 6th Framework Programme MRTN-CT-2004-503369. J.F. has been supported by the research fellowship MIUR PRIN 2004 - "Astroparticle Physics". K.O. has been supported by the grant-in-aid for scientific research on priority areas (No. 441): "Progress in elementary particle physics of the 21st century through discoveries of Higgs boson and supersymmetry" (No. 16081209) from the Ministry of Education, Culture, Sports, Science and Technology of Japan and the Korean government grant KRF PBRG 2002-070-C00022. L.R. would like to thank A. Masiero and INFN Padova for kind hospitality during his recent visit when this work was finalised.

\section{Appendix}

The factors of $\epsilon$ that enter into Eq. (10) have the form [4]

$$
\begin{aligned}
\epsilon_{s} & =-\frac{\alpha_{s}}{2 \pi} C_{2}(3) \frac{\mu}{m_{\widetilde{g}}} H_{2}\left(x_{\widetilde{d}_{R}}, x_{\widetilde{d}_{L}}\right), & \epsilon_{Y} & =-\frac{A_{u}}{16 \pi^{2} \mu} H_{2}\left(y_{\widetilde{u}_{R}}, y_{\widetilde{u}_{L}}\right), \\
\epsilon_{R L} & =-\frac{\alpha_{s}}{2 \pi} C_{2}(3) H_{2}\left(x_{\widetilde{d}_{R}}, x_{\widetilde{d}_{L}}\right), & \epsilon_{L L} & =-\frac{\alpha_{s}}{2 \pi} C_{2}(3) \frac{\mu}{m_{\widetilde{g}}} H_{3}\left(x_{\widetilde{d}_{R}}, x_{\widetilde{d}_{R}}, x_{\widetilde{d}_{L}}\right), \\
\epsilon_{3} & =\epsilon_{s}+\epsilon_{Y} Y_{t}^{2} & &
\end{aligned}
$$

where $\alpha_{s}$ denotes the strong coupling constant, $C_{2}(3)=4 / 3$ is the quadratic Casimir for SU(3) and $A_{u}$ is related to the soft terms $m_{u, L R}^{2}$ via the relation $\left(m_{u, L R}^{2}\right)=A_{u} m_{u}$. The loop functions that appear in the above expressions are

$$
\begin{aligned}
H_{2}\left(x_{1}, x_{2}\right) & =\frac{x_{1} \log x_{1}}{\left(1-x_{1}\right)\left(x_{1}-x_{2}\right)}+\frac{x_{2} \log x_{2}}{\left(1-x_{2}\right)\left(x_{2}-x_{1}\right)}, \\
H_{3}\left(x_{1}, x_{2}, x_{3}\right) & =\frac{H_{2}\left(x_{1}, x_{2}\right)-H_{2}\left(x_{1}, x_{3}\right)}{x_{2}-x_{3}} .
\end{aligned}
$$

Finally, the arguments of the various loop functions are given by

$$
x_{\widetilde{d}_{L}}=\frac{m_{d, L L}^{2}}{m_{\widetilde{g}}^{2}}, \quad y_{\widetilde{u}_{L}}=\frac{m_{u, L L}^{2}}{\mu^{2}}, \quad x_{\widetilde{d}_{R L}}=\frac{\sqrt{m_{d, L L}^{2} m_{d, R R}^{2}}}{m_{\widetilde{g}}^{2}} .
$$

The remaining factors of $\epsilon, x$ and $y$ may be obtained by the appropriate generalisations of the above expressions. Note that the soft terms that appear in the above equation are the common values of the diagonal entries of the SUSY soft terms. 


\section{References}

[1] For instance see:

A. J. Buras, P. Gambino, M. Gorbahn, S. Jager and L. Silvestrini, Phys. Lett. B 500 (2001) 161 arXiv:hep-ph/0007085;

G. D'Ambrosio, G. F. Giudice, G. Isidori and A. Strumia, Nucl. Phys. B 645, 155 (2002) arXiv:hep-ph/0207036;

C. Bobeth, M. Bona, A. J. Buras, T. Ewerth, M. Pierini, L. Silvestrini and A. Weiler, Nucl. Phys. B 726 (2005) 252 arXiv:hep-ph/0505110.

[2] F. Gabbiani, E. Gabrielli, A. Masiero and L. Silvestrini, Nucl. Phys. B 477 (1996) 321 arXiv:hep-ph/9604387.

[3] K. Okumura and L. Roszkowski, Phys. Rev. Lett. 92 (2004) 161801 arXiv:hep-ph/0208101;

K. Okumura and L. Roszkowski, JHEP 0310 (2003) 024 arXiv:hep-ph/0308102.

[4] J. Foster, K. Okumura and L. Roszkowski, Phys. Lett. B 609 (2005) 102 arXiv:hep-ph/0410323;

J. Foster, K. Okumura and L. Roszkowski, JHEP 0508 (2005) 094 arXiv:hep-ph/0506146.

[5] J. Foster, K. Okumura and L. Roszkowski, JHEP 0603 (2006) 044 arXiv:hep-ph/0510422.

[6] DØ Collaboration, "A Study of $B_{s}^{0}$ Oscillations," DØ note 5045-CONF;

V. Abazov [D $\varnothing$ Collaboration], arXiv:hep-ex/0603029.

[7] G. Gomez-Ceballos [CDF Collaboration], Talk given at FPCP 2006, http://fpcp2006.triumf .ca/talks/day3/1500/fpcp2006.pdf

CDF Collaboration, http://www-cdf .fnal.gov/physics/new/bottom/060406.blessed-Bsmix/.

[8] J. Charles et al. [CKMfitter Group], Eur. Phys. J. C 41 (2005) 1 arXiv:hep-ph/0406184 http://ckmfitter.in2p3.fr

M. Bona et al. [UTfit Collaboration], JHEP 0603 (2006) 080 arXiv:hep-ph/0509219 http://utfit.roma1.infn.it/.

[9] A. J. Buras, M. Jamin and P. H. Weisz, Nucl. Phys. B 347 (1990) 491.

[10] P. Ball and R. Fleischer, arXiv:hep-ph/0604249

[11] S. Hashimoto, Int. J. Mod. Phys. A 20 (2005) 5133 arXiv:hep-ph/0411126.

[12] S. Bertolini, F. Borzumati, A. Masiero and G. Ridolfi, Nucl. Phys. B 353 (1991) 591;

[13] C. Hamzaoui, M. Pospelov and M. Toharia, Phys. Rev. D 59 (1999) 095005 arXiv:hep-ph/9807350;

P. H. Chankowski and E. Sławianowska, Phys. Rev. D 63 (2001) 054012 arXiv:hep-ph/0008046;

G. Isidori and A. Retico, JHEP 0209 (2002) 063 arXiv:hep-ph/0208159.

[14] S. R. Choudhury and N. Gaur, Phys. Lett. B 451 (1999) 86 arXiv:hep-ph/9810307;

K. S. Babu and C. F. Kolda, Phys. Rev. Lett. 84, 228 (2000) arXiv:hep-ph/9909476;

C. S. Huang, W. Liao, Q. S. Yan and S. H. Zhu, Phys. Rev. D 63 (2001) 114021 [Erratum-ibid.

D 64 (2001) 059902] arXiv:hep-ph/0006250; 
C. Bobeth, T. Ewerth, F. Kruger and J. Urban, Phys. Rev. D 64 (2001) 074014 arXiv:hep-ph/0104284;

G. Isidori and A. Retico, JHEP 0111 (2001) 001 arXiv:hep-ph/0110121

C. Bobeth, T. Ewerth, F. Kruger and J. Urban, Phys. Rev. D 66 (2002) 074021 arXiv:hep-ph/0204225.

[15] A. J. Buras, P. H. Chankowski, J. Rosiek and Ł. Sławianowska, Nucl. Phys. B 619 (2001) 434 arXiv:hep-ph/0107048;

A. J. Buras, P. H. Chankowski, J. Rosiek and E. Sławianowska, Phys. Lett. B 546 (2002) 96 arXiv:hep-ph/0207241;

A. J. Buras, P. H. Chankowski, J. Rosiek and E. Sławianowska, Nucl. Phys. B 659 (2003) 3 arXiv:hep-ph/0210145.

[16] A. Dedes and A. Pilaftsis, Phys. Rev. D 67, 015012 (2003) arXiv:hep-ph/0209306;

M. Carena, A. Menon, R. Noriega-Papaqui, A. Szynkman and C. E. M. Wagner, arXiv:hep-ph/0603106.

[17] T. Besmer, C. Greub and T. Hurth, Nucl. Phys. B 609 (2001) 359 arXiv:hep-ph/0105292.

[18] CDF Collaboration, "Search for FCNC Rare Decay $B_{s} \rightarrow \mu^{+} \mu^{-}$," CDF note 8176.

[19] G. Buchalla and A. J. Buras, Nucl. Phys. B 398 (1993) 285

G. Buchalla and A. J. Buras, Nucl. Phys. B 400 (1993) 225;

M. Misiak and J. Urban, Phys. Lett. B 451 (1999) 161 arXiv:hep-ph/9901278.

G. Buchalla and A. J. Buras, Nucl. Phys. B 548 (1999) 309 arXiv:hep-ph/9901288.

[20] G. Degrassi, P. Gambino and G. F. Giudice, JHEP 0012 (2000) 009 arXiv:hep-ph/0009337;

M. Carena, D. Garcia, U. Nierste and C. E. M. Wagner, Phys. Lett. B 499 (2001) 141 arXiv:hep-ph/0010003.

[21] T. Banks, Nucl. Phys. B 303 (1988) 172;

R. Hempfling, Phys. Rev. D 49, 6168 (1994);

L. J. Hall, R. Rattazzi and U. Sarid, Phys. Rev. D 50 (1994) 7048 arXiv:hep-ph/9306309;

M. Carena, M. Olechowski, S. Pokorski and C. E. M. Wagner, Nucl. Phys. B 426 (1994) 269 arXiv:hep-ph/9402253;

T. Blazek, S. Raby and S. Pokorski, Phys. Rev. D 52 (1995) 4151 arXiv:hep-ph/9504364.

[22] M. Carena, D. Garcia, U. Nierste and C. E. M. Wagner, Nucl. Phys. B 577 (2000) 88 arXiv:hep-ph/9912516.

[23] M. Blanke, A. J. Buras, D. Guadagnoli and C. Tarantino, arXiv:hep-ph/0604057,

Z. Ligeti, M. Papucci and G. Perez, arXiv:hep-ph/0604112.

[24] M. Ciuchini and L. Silvestrini, arXiv:hep-ph/0603114;

M. Endo and S. Mishima, arXiv:hep-ph/0603251.

[25] S. Khalil, arXiv:hep-ph/0605021;

Y. Grossman, Y. Nir and G. Raz, arXiv:hep-ph/0605028

A. Datta, arXiv:hep-ph/0605039

[26] E. Barberio et al. [The Heavy Flavor Averaging Group], arXiv:hep-ex/0603003 
[27] P. Gambino and M. Misiak, Nucl. Phys. B 611 (2001) 338 arXiv:hep-ph/0104034;

A. J. Buras, A. Czarnecki, M. Misiak and J. Urban, Nucl. Phys. B 631 (2002) 219 arXiv:hep-ph/0203135.

[28] A. J. Buras, S. Jager and J. Urban, Nucl. Phys. B 605 (2001) 600 arXiv:hep-ph/0102316.

[29] S. Baek, T. Goto, Y. Okada and K. Okumura, Phys. Rev. D 63 (2001) 051701 arXiv:hep-ph/0002141;

T. Moroi, JHEP 0003 (2000) 019 arXiv:hep-ph/0002208;

T. Moroi, Phys. Lett. B 493 (2000) 366 arXiv:hep-ph/0007328;

S. Baek, T. Goto, Y. Okada and K. Okumura, Phys. Rev. D 64 (2001) 095001 arXiv:hep-ph/0104146;

D. Chang, A. Masiero and H. Murayama, Phys. Rev. D 67 (2003) 075013 arXiv:hep-ph/0205111. 